\title{
Revisión del uso de los medicamentos (RUM), un nuevo servicio profesional en la farmacia comunitaria española
}

\author{
Vicente J. Baixauli \\ Vicepresidente de SEFAC y coordinador de la Comisión del servicio RUM de SEFAC.
}

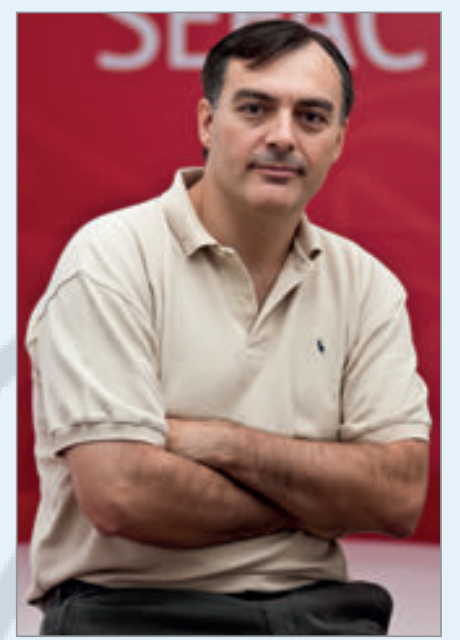

Vicente J. Baixauli
Si hay una actividad transversal a casi todos los servicios profesionales farmacéuticos asistenciales relacionados con los medicamentos, esa es la revisión de los medicamentos o revisión de la farmacoterapia. Desde una revisión de botiquín hasta el seguimiento farmacoterapéutico pasando por la dispensación, la conciliación o la indicación farmacéutica, en todos estos servicios se realiza una revisión de los medicamentos que utiliza el paciente.

Sin embargo, la revisión de los medicamentos se puede realizar a tres niveles según la clasificación de la Pharmaceutical Care Network Europe (PCNE) que son acumulativos. El primer nivel lo constituye la revisión de las prescripciones realizadas por el médico. El segundo se corresponde con una revisión realizada cara a cara con el paciente y los medicamentos que utiliza, y la tercera sería una revisión clínica más completa en la que se dispone de todos los datos clínicos del paciente. Sin embargo, el servicio de Revisión del Uso de los Medicamentos (RUM) es un servicio que no se ajusta a ninguno de estos niveles como tal, pero que se asemeja a una revisión tipo 2 centrada únicamente en el proceso de uso de los medicamentos por el paciente.

Tras los programas de capacitación: impacHta (presión arterial y riesgo vascular), CESAR (cesación tabáquica), EPOCA (EPOC) y DIFAC (diabetes), llega Revisa o, lo que es lo mismo, el programa para la implantación en nuestro país del servicio RUM. El servicio de RUM es el primer servicio profesional farmacéutico que desarrolla SEFAC centrado exclusivamente en los medicamentos y que se protocoliza para la farmacia comunitaria en España como tal. Un servicio de RUM consiste en la realización por el farmacéutico junto con el paciente (o cuidador) de una revisión estructurada del grado de conocimiento que éste tiene de sus medicamentos y del uso que hace de los mismos, ofreciendo asesoramiento sobre su utilización correcta y asegurándose de que el paciente entiende por qué los tiene que tomar y sabe cómo utilizarlos y/o administrarlos. Además, es necesario identificar los problemas observados y realizar las recomendaciones oportunas sobre los cambios necesarios $\mathrm{y}$, en su caso, comunicándolos al médico prescriptor mediante un formulario específico. El objetivo general del servicio de RUM es que los pacientes mejoren el conocimiento, la adherencia y el proceso de uso de los medicamentos. Este servicio viene a complementar al resto de servicios, ya que engarza muy bien con el servicio de dispensación, seguimiento farmacoterapéutico, indicación farmacéutica, preparación de sistemas personalizados, educación sanitaria, medición y control de la presión arterial y el riesgo vascular, cesación tabáquica, etc.

El servicio de RUM es una adaptación a la farmacia comunitaria española del servicio profesional farmacéutico retribuido como es el Medication Use Review (MUR) británico. Esta adaptación se ha realizado a través de una comisión en la que hay varios compañeros que o bien habían estado trabajando en el Reino Unido en los últimos años o bien están trabajando allí como farmacéuticos comunitarios y están capacitados para la prestación del servicio MUR. Todos ellos, junto al resto de miembros de la comisión de RUM de SEFAC, han elaborado también el documento de especificaciones del servicio de RUM y su procedimiento normalizado de trabajo. Ambos documentos fueron presentados en la jornada de servicios profesionales realizada en noviembre de 2013 en Barcelona. 
A partir de entonces se comenzó a trabajar en el diseño de un estudio piloto y en un programa de capacitación para este servicio.

Todas estas iniciativas, que tienen como finalidad la implantación del servicio de RUM en la farmacia comunitaria española, constituyen el proyecto Revisa.

La determinación de llevar adelante este proyecto siempre ha sido clara. No en vano, este servicio aporta numerosos beneficios, tanto para los pacientes como para los farmacéuticos y, por extensión, para el propio sistema sanitario. En primer lugar, es evidente que existe un problema claro con el uso de los medicamentos y la adherencia terapéutica, no solo en España sino a nivel mundial. Trabajos como el $D$-VALOR en nuestro país han puesto de manifiesto que más de un 43\% de la población tiene carencias informativas significativas sobre sus tratamientos (se desconoce la posología, duración, etc.); además, la Organización Mundial de la Salud (OMS) estima que más del 50\% de los medicamentos no se toma correctamente y hasta un tercio de las visitas a los servicios de urgencias es consecuencia de un resultado no deseado del medicamento, cuando más del 70\% de estos resultados no deseados son evitables.

Si el farmacéutico comunitario tuviera un mayor protagonismo en la gestión de los tratamientos y colaborara con los médicos en las tareas de revisión de los medicamentos se podrían solventar mejor los problemas de falta de adherencia y polimedicación. Esto supone un beneficio claro para la salud de los pacientes (en especial para los polimedicados y para aquellos que utilizan medicamentos especialmente complejos o con patologías de alto riesgo) pero también para la sostenibilidad del sistema, pues proporciona una mayor garantía en el uso adecuado y seguro de los medicamentos y de los recursos destinados a su financiación por la Administración.

Por si esto fuera poco, el RUM eleva la consideración profesional del farmacéutico comunitario, pues le permite un mejor conocimiento sobre el uso de la farmacoterapia del paciente, le posibilita una oportunidad de realizar educación sanitaria, mejora sus habilidades clínicas y de educación para la salud y facilita una mejor relación con otros profesionales de la salud. Sin olvidar que este servicio, en el Reino Unido, está remunerado y es uno de los mejor valorados por la población y el National Health Service (NHS).

En definitiva, con el RUM se consigue:

- Mejorar del conocimiento, la adherencia y el proceso de uso de los medicamentos que siguen los pacientes.

- Averiguar el conocimiento, el uso real, la comprensión sobre su utilización y la experiencia del paciente al tomar sus medicamentos.

- Identificar, examinar y resolver el mal uso o uso ineficaz de los medicamentos a través de la detección de los problemas relacionados con los medicamentos (PRM) (administración errónea del medicamento, dosis, pauta y/o duración no adecuada, interacciones, etc.) y resultados negativos de los medicamentos (RNM) (efectos secundarios), que inciden sobre la adherencia al tratamiento farmacológico del paciente.

Por todo ello, en 2016 está previsto iniciar el estudio piloto de Revisa, ya clasificado por la Agencia Española de Medicamentos y Productos Sanitarios (AEMPS) y en el que participarán 76 farmacéuticos repartidos por toda España que se capacitarán para prestar este servicio (previo paso por una formación online y presencial específica y el registro de casos clínicos). Los resultados preliminares de este proyecto se presentarán en el VII Congreso Nacional de Farmacéuticos Comunitarios que se celebrará en Zaragoza el próximo mes de mayo de 2016 y a partir de septiembre de 2016 el resto de farmacéuticos españoles tendrán a su disposición un nuevo servicio farmacéutico en el que capacitarse y prestar a los pacientes en sus farmacias.

La llegada del RUM a España será una magnífica oportunidad para que los farmacéuticos comunitarios podamos desarrollar, por fin, un servicio profesional sobre el que nadie podrá poner en duda la idoneidad de llevarlo a cabo en nuestras farmacias. Un servicio hecho en la farmacia comunitaria por el principal experto en medicamentos y el más cercano a la población: el farmacéutico comunitario. 